\title{
Self-Monitoring and Management of Blood Pressure in Patients with Stroke or TIA: An Economic Evaluation of TEST-BP, A Randomised Controlled Trial
}

\author{
Lois G. Kim $^{1,2}$ - Edward C. F. Wilson ${ }^{1,3}$ (D) William J. Davison ${ }^{4}$ - Allan B. Clark ${ }^{5}$ Phyo K. Myint ${ }^{6}$. John F. Potter ${ }^{4}$
}

Published online: 13 February 2020

(c) The Author(s) 2020

\begin{abstract}
Background Prevention of secondary stroke following initial ictus is an important focus of after-stroke care. Blood pressure (BP) is a key risk factor, so usual care following stroke or transient ischaemic attack includes regular BP checks and monitoring of anti-hypertensive medication. This is traditionally carried out in primary care, but the evidence supporting self-monitoring and self-guided management of BP in the general population with hypertension is growing.

Objective Our objective was to estimate the cost effectiveness of treatment as usual (TAU) versus (1) self-monitoring of BP (S-MON) and (2) self-monitoring and guided self-management of anti-hypertensive medication (S-MAN).

Methods This was a within-trial economic evaluation of a randomised controlled trial estimating the incremental cost per $1 \mathrm{mmHg}$ BP reduction and per quality-adjusted life-year (QALY) gained over a 6-month time horizon from the perspective of the UK National Health Service (NHS).

Results Data were evaluable for 140 participants. Costs per patient were $£ 473$, $£ 853$ and $£ 1035$; mean reduction in systolic BP (SBP) was 3.6, 6.7 and $6.1 \mathrm{mmHg}$, and QALYs accrued were $0.427,0.422$ and 0.423 for TAU, S-MON and S-MAN, respectively. No statistically significant differences in incremental costs or outcomes were detected. On average, S-MAN was dominated or extended dominated. The incremental cost per $1 \mathrm{mmHg}$ BP reduction from S-MON versus TAU was £137. Conclusion On average, S-MAN is an inefficient intervention. S-MON may be cost effective, depending on the willingness to pay for a $1 \mathrm{mmHg}$ BP reduction, although it yielded fewer QALYs over the within-trial time horizon. Decision modelling is required to explore the longer-term costs and outcomes.
\end{abstract}

Electronic supplementary material The online version of this article (https://doi.org/10.1007/s41669-020-00196-w) contains supplementary material, which is available to authorized users.

Edward C. F. Wilson

ed.wilson@uea.ac.uk

1 Cambridge Centre for Health Services Research, Institute of Public Health, University of Cambridge School of Clinical Medicine, Forvie Site, Cambridge, UK

2 Cardiovascular Epidemiology Unit, Department of Public Health and Primary Care, Strangeways Research Laboratory, Wort's Causeway, Cambridge, UK

3 Health Economics Group, Norwich Medical School, University of East Anglia, Norwich, UK

4 Ageing and Stroke Medicine, Norwich Medical School, University of East Anglia, Norwich, UK

5 Medical Statistics Group, Norwich Medical School, University of East Anglia, Norwich, UK

6 Ageing Clinical and Experimental Research (ACER), Institute of Applied Health Sciences, University of Aberdeen, Aberdeen, UK

\section{Introduction}

Prevention of stroke recurrence following initial stroke or transient ischaemic attack (TIA) is an important focus for secondary prevention, the risk being substantially reduced by good blood pressure (BP) control [1-3]. Usual care following stroke and TIA therefore includes regular BP checks and monitoring of anti-hypertensive medications, which are usually carried out in primary care.

In recent years, evidence supporting the use of selfmonitoring and guided management of BP in the general population with high BP has been growing $[4,5]$. Several studies have reported benefits in terms of BP reductions and improved control, and this approach therefore offers a potential opportunity to not only translate these benefits to patients with a history of stroke or TIA but also to reduce the primary care burden associated with after-stroke care. Since no consensus yet exists regarding whether specific subgroups (such as those with a history of stroke/TIA) benefit 


\section{Key Points for Decision Makers}

On average, over a 6-month time horizon, self-management of anti-hypertensive medication is not an efficient intervention. The incremental cost per $1 \mathrm{mmHg}$ blood pressure (BP) reduction for self-monitoring of BP versus treatment as usual (TAU) was $£ 137$.

Point estimate quality-adjusted life-years (QALYs) accrued were very similar, although TAU had the highest point estimate.

As future cardiovascular events will be a major driver of QALYs, decision modelling is required to explore longer-term costs and outcomes.

from this strategy, or whether guided self-management in conjunction with self-monitoring offers a superior approach to self-monitoring alone [6], it remains important to evaluate these strategies in specific subpopulations of interest.

TEST-BP (Trial of the Effectiveness of Self-monitoring/ Treatment of BP after stroke; ClinicalTrials.gov reference NCT02947490) was a randomised controlled trial that assessed the benefits in terms of BP reduction and reaching target levels using these approaches in patients following stroke or TIA. The trial reported small and statistically nonsignificant improvements in systolic BP (SBP) over 6 months in patients using both self-monitoring of $\mathrm{BP}$ and self-monitoring plus guided management of their anti-hypertensive medications compared with usual care [7]. Although BP reduction and control rates were not statistically significantly different between the interventions, these results demonstrated that this approach to after-stroke BP care was both safe and well-tolerated (only $2 \%$ of patients receiving the intervention did not comply with the BP measurement protocol) among patients with stroke or TIA. Self-monitoring and guided self-management were associated with significantly greater increases in anti-hypertensive drug use than was usual treatment.

In this study, we estimated the within-trial cost effectiveness of self-monitoring and self-management of BP in patients with a history of stroke or TIA. We used patient data from TEST-BP to combine information about the SBP reduction benefits and the associated costs to assess the short-term cost effectiveness of these interventions.

\section{Methods}

\subsection{Clinical Study}

Full details of the results of TEST-BP have been published elsewhere [7]. In brief, 171 community-dwelling adults in
England with a recent mild or moderate stroke or TIA requiring anti-hypertensive treatment to prevent recurrence were randomised into the three arms of the trial between December 2012 and March 2016, of whom 154 were included in the final outcomes analysis: (1) treatment as usual (TAU, $n=52$ ), (2) self-monitoring of BP (S-MON, $n=51$ ), (3) self-monitoring of BP plus guided management of antihypertensive medications (S-MAN, $n=51$ ). A target BP of $\leq 130 / 80 \mathrm{mmHg}$ was used for office measurement, adjusted downwards to $120 / 75 \mathrm{mmHg}$ for out-of-office measurement in the intervention groups (S-MON, S-MAN). This was to counteract the so-called 'white coat' effect of BP measurement [8]. Daytime ambulatory BP monitoring values were used to provide BP measurements (study primary outcome) at baseline and 6-month follow-up. The intervention for both S-MON and S-MAN groups comprised self-monitoring over a 7-day period, taking two readings in the morning before treatment and two again about $12 \mathrm{~h}$ later using validated monitors at 6 weeks, 3 months and 5 months after baseline. Self-recorded values were passed to the general practitioner (GP) in the S-MON group by post/telephone and directly to the study team in the S-MAN group by telemetry, with a paper copy to the patient's GP; the S-MAN group then received guidance from the study clinician regarding possible adjustments to their medications and jointly agreed on any changes without the need for a face-to-face appointment with their GP. Medication management in the TAU and S-MON groups was carried out by the GP with no input from the trial team. Data on changes in treatment, GP visits and adverse events were recorded at each study visit in TAU and S-MON at the beginning and end of the trial.

\subsection{Health Economic Measurements and Analysis}

Profiles as per the three-level EuroQoL-Five Dimensions (EQ-5D-3L) instrument were collected at baseline and 6-month follow-up and converted to utilities using UKspecific preference weights [9]. Quality-adjusted life-years (QALYs) over the follow-up period were then calculated as the area under the curve using linear interpolation between the two timepoints. Comparisons of QALYs between groups were adjusted for baseline utility [10].

Costs were calculated by extracting resource-use quantities from the patient-completed resource-use questionnaires and multiplying them by unit costs taken from standard national health service (NHS) sources for 2017/18 [11, 12]. Unit costs are reported in the online appendix. Where appropriate, costs were adjusted to year $2017 / 8$ values using the hospital and community health services pay and prices index [13] (to 2016) and the new health services index [11]. Anti-hypertensive medication costs were calculated using frequency and dose of prescription alongside data on pack 
size and cost relevant for the NHS [14, 15]. To minimise respondent burden, a maximum of one medication per category ( $\alpha$-blockers, $\beta$-blockers, calcium channel blockers, diuretics, angiotensin-converting enzyme inhibitors or angiotensin-receptor blockers, and other anti-hypertensive medications) was recorded at baseline, 6 weeks, 3 months, and 5 months and at 6-month follow-up, with usage assumed constant between timepoints. The cost of the intervention included purchase price of the BP monitor and associated software costs, two nurse home visits incorporating time spent training patients in their use (nurse time and mileage costs applied) and telephone charges to remind patients to monitor their BP and to discuss treatment changes in the S-MON and S-MAN arms [11]. Prices for monitors and associated software ( $€$, year $2012 / 3$ values) were converted to $£$ at the historic rate (2012) of $€ 1$ : $£ 0.833$, before being adjusted to year 2018 values. The economic evaluation was conducted from the perspective of the NHS over a time horizon of 6 months (within-trial evaluation). No discounting was applied because of the short time horizon of the study. Data were analysed with Stata v16.0 (Stata Corp LLC, College Station, TX, USA).

We report mean (95\% confidence interval [CI]) change in SBP from baseline, QALYs accrued and cost per patient, with increments $\pm 95 \%$ CI reported compared with TAU. Primary analysis was based on as-treated groups; a sensitivity analysis was then carried out based on intention to treat (ITT). Point estimate incremental cost-effectiveness ratios (ICERs) are reported taking into account dominance and extended dominance, with uncertainty in the ICERs reported via cost-effectiveness acceptability curves (CEACs) and frontiers, showing the probability that each of the interventions is the most cost effective at various thresholds of willingness to pay (WTP) per point reduction in SBP. CEACs were calculated using 10,000 bootstrapped samples of the dataset. As the quantity of missing data was relatively low (approximately 10\%; see Sect. 3), we report complete case analysis only (i.e. no adjustments for missing data).

\section{Results}

We excluded 14 participants (as-treated groups: one TAU, eight S-MON, five S-MAN) from the economic evaluation because their care resource-use data were incomplete. In total, 140 participants were analysed (51 TAU, 43 S-MON, 46 S-MAN), with a further five patients (two TAU, two S-MON, one S-MAN) excluded from the QALY analysis because of missing utility data.

Table 1 shows the mean resource use and costs in each group, categorised by costs relating directly to the invention, primary/community care use, secondary care use and antihypertensive medications. Mean overall costs were $£ 473$ for
TAU, $£ 853$ for S-MON and $£ 1035$ for S-MAN. Differences were not statistically significant (all $p \geq 0.2$ ).

Table 2 presents the key outcomes relating to the economic evaluation. The incremental cost per additional $1 \mathrm{mmHg}$ SBP reduction with S-MON compared with TAU was £137. S-MON dominated S-MAN (i.e. on average, it was both more effective and less expensive). Figure 1 shows the net benefit for each randomised group over a range of WTP values per $1 \mathrm{mmHg}$ SBP reduction, evaluated at the mean baseline SBP. This suggests that TAU is the preferred approach at WTP values $<£ 137$ for $1 \mathrm{mmHg}$ SBP reduction; at higher values, S-MON is the preferred approach. Figure 2 shows the corresponding CEACs. For the ITT analysis, S-MON had the highest probability of cost effectiveness as long as the WTP for a $1 \mathrm{mmHg}$ SBP reduction was $>£ 196$. For the analysis based on QALYs, TAU dominated both the S-MON and the S-MAN treatment strategies. Figures 3 and 4 show the net benefit and CEAC plots, respectively, corresponding to this analysis: TAU was the most cost-effective option, with very high certainty over this limited timeframe.

\section{Discussion}

In terms of BP reduction, on average, S-MON cost $£ 137$ per $1 \mathrm{mmHg}$ SBP reduction over 6 months compared with TAU. When taking an ITT approach, the ICER was $£ 196$ per $1 \mathrm{mmHg}$ SBP reduction. S-MAN was not an efficient option, yielding poorer outcomes and higher costs than S-MON. This was mostly due to the increased intervention cost, but the higher point estimate cost of anti-hypertensives is worth noting: the S-MAN group tended to use larger doses of medication and more different agents than the S-MON group, but these did not result in better BP control (at least within the 6-month trial time horizon) [7]. When considering cost effectiveness in terms of QALYs, TAU dominated both S-MON and S-MAN because point estimate QALYs were lower than TAU. However, we urge caution in interpreting these results: QALY accruals will be driven much more strongly by future cardiovascular events, which cannot be captured within a 6-month time horizon. Combining our results with longer-term prognoses may yield increased QALYs through reduced BP, but decision modelling is required to confirm or refute this.

TEST-BP was restricted to the post-stroke and TIA subpopulation, so may be expected to provide slightly different estimates of the cost effectiveness of these interventions than studies carried out in other populations. One randomised trial of self-monitoring of BP compared with usual care in the wider hypertensive population reported that self-monitoring was cheaper because of reduced medication costs [16]. However, in that study, the self-monitoring target BP was not adjusted down, 
Table 1 Mean resource use and associated cost by group, over the 6-month follow-up period (for those not lost to follow-up)

\begin{tabular}{|c|c|c|c|c|c|c|}
\hline \multirow[t]{2}{*}{ Intervention } & \multicolumn{2}{|c|}{ TAU $(N=51)$} & \multicolumn{2}{|c|}{ S-MON $(N=43)$} & \multicolumn{2}{|c|}{ S-MAN $(N=46)$} \\
\hline & Contacts & Cost & Contacts & Cost & Contacts & Cost \\
\hline Total cost & & 0 & & 185 & & 379 \\
\hline \multicolumn{7}{|l|}{ Primary and community care } \\
\hline GP-surgery & $1.5 \pm 1.4$ & $56 \pm 51$ & $1.8 \pm 2.4$ & $67 \pm 89$ & $1.6 \pm 2.4$ & $59 \pm 87$ \\
\hline GP-phone & $0.02 \pm 0.1$ & $0.16 \pm 1.13$ & $0.2 \pm 0.5$ & $1.32 \pm 4.30$ & $0.1 \pm 0.5$ & $0.88 \pm 3.90$ \\
\hline GP-home & 0 & NA & 0 & NA & 0 & $0 \pm 0$ \\
\hline Nurse-surgery & $0.8 \pm 0.9$ & $9.15 \pm 9.79$ & $1.3 \pm 4.1$ & $14.13 \pm 44.01$ & $0.6 \pm 1.6$ & $6.60 \pm 17.04$ \\
\hline Nurse-phone & $0.02 \pm 0.1$ & $0.10 \pm 0.70$ & 0 & NA & 0 & $0 \pm 0$ \\
\hline Nurse-home & 0 & NA & $0.8 \pm 4.6$ & $9.08 \pm 50.39$ & 0 & $0 \pm 0$ \\
\hline Health visitor-surgery & 0 & NA & 0 & NA & 0 & $0 \pm 0$ \\
\hline Health visitor-phone & 0 & NA & 0 & NA & 0 & $0 \pm 0$ \\
\hline Health visitor-home & 0 & NA & 0 & NA & $0.1 \pm 0.9$ & $6.72 \pm 45.60$ \\
\hline Physiotherapist—surgery & $0.2 \pm 1.4$ & $12.13 \pm 72.60$ & $0.2 \pm 1.0$ & $9.59 \pm 49.36$ & $0.2 \pm 1.5$ & $11.21 \pm 76.01$ \\
\hline Physiotherapist-phone & 0 & NA & 0 & NA & 0 & $0 \pm 0$ \\
\hline Physiotherapist-home & 0 & NA & $0.05 \pm 0.3$ & $2.40 \pm 15.72$ & $0.2 \pm 0.9$ & $10.09 \pm 48.14$ \\
\hline OT-surgery & $0.3 \pm 1.5$ & $14.15 \pm 77.21$ & 0 & NA & 0 & $0 \pm 0$ \\
\hline OT-phone & 0 & NA & 0 & NA & 0 & $0 \pm 0$ \\
\hline OT-home & $0.02 \pm 0.1$ & $1.01 \pm 7.22$ & $0.05 \pm 0.3$ & $2.40 \pm 15.72$ & $0.02 \pm 0.1$ & $1.12 \pm 7.60$ \\
\hline Other-surgery & $0.3 \pm 1.5$ & $16.17 \pm 76.11$ & $0.07 \pm 0.3$ & $3.60 \pm 17.41$ & $0.2 \pm 0.9$ & $8.97 \pm 46.50$ \\
\hline Other-phone & 0 & NA & 0 & NA & 0 & $0 \pm 0$ \\
\hline Other-home & $0.04 \pm 0.2$ & $2.02 \pm 10.11$ & 0 & NA & 0 & $0 \pm 0$ \\
\hline Total cost & & $111 \pm 242$ & & $110 \pm 157$ & & $104 \pm 181$ \\
\hline \multicolumn{7}{|l|}{ Secondary care } \\
\hline Outpatient appointment & $0.6 \pm 1.4$ & $76 \pm 172$ & $0.9 \pm 1.6$ & $108 \pm 194$ & $0.7 \pm 2.3$ & $90 \pm 285$ \\
\hline Day-case procedure & $0.1 \pm 0.5$ & $73 \pm 340$ & $0.07 \pm 0.3$ & $52 \pm 191$ & $0.1 \pm 0.7$ & $81 \pm 547$ \\
\hline Inpatient admission & $0.06 \pm 0.2$ & $195 \pm 787$ & $0.1 \pm 0.4$ & $385 \pm 1294$ & $0.1 \pm 0.5$ & $360 \pm 1596$ \\
\hline Total cost & & $343 \pm 948$ & & $544 \pm 1458$ & & $530 \pm 1919$ \\
\hline \multicolumn{7}{|l|}{ Anti-hypertensive medication } \\
\hline Total cost & & $19 \pm 27$ & & $14 \pm 19$ & & $21 \pm 26$ \\
\hline Grand total cost & & $473 \pm 1045$ & & $853 \pm 1458$ & & $1035 \pm 1953$ \\
\hline
\end{tabular}

Data are presented as mean \pm standard deviation; costs are presented as $£$

$G P$ general practitioner, $N A$ not applicable, $O T$ occupational therapist, $S$-MAN self-monitoring and guided self-management of anti-hypertensive medication, $S$-MON self-monitoring of blood pressure, $T A U$ treatment as usual

which may have influenced treatment costs. Furthermore, primary and secondary care costs were not included, and details on the methods used to compare costs between groups were not given. A systematic review of self-monitoring with telemonitoring by Omboni et al. [17], again in the wider hypertensive population, incorporated a cost-effectiveness analysis. Six studies were analysed, and the authors reported that the cost of selfmonitoring with telemonitoring was greater than that of usual care by around $€ 660$, with a cost per $1 \mathrm{mmHg}$ SBP reduction of $€ 406$. However, there was substantial heterogeneity between the studies, with only three reporting on medical costs in addition to intervention costs, and a wide range of follow-up durations. Along with the difference in population studied, this likely accounts for the differences from our findings.
A key strength of our study was the use of ambulatory BP monitoring for the outcome measure, as opposed to clinic BP measurement used in other studies [18, 19], thus decreasing the influence of the natural variability in BP measurement and reducing the risk of measurement bias [20]. Another strength was the simultaneous comparison of two intervention strategies, this being the first health economic evaluation of guided self-management exclusively in a stroke/TIA population.

The major limitation of this study was that it only provided a short-term perspective on the benefits and costs associated with these interventions, focusing on the impact on BP. Studies with durations $>24$ months have shown that the benefits of BP self-monitoring in terms of control decreased with time [21]. However, even modest BP reductions may 
Table 2 Key costs and outcomes results for economic evaluation

\begin{tabular}{|c|c|c|c|}
\hline Costs and outcomes & TAU & S-MON & S-MAN \\
\hline Primary analysis: SBP & $N=51$ & $N=43$ & $N=46$ \\
\hline Mean reduction in SBP & $3.6(0.2$ to 7.0$)$ & $6.7(3.1$ to 10.4$)$ & $6.1(2.6$ to 9.7$)$ \\
\hline Incremental reduction in $\mathrm{SBP}^{\mathrm{a}, \mathrm{b}}$ & NA & $2.8(-1.6$ to 7.1$)$ & $2.7(-1.5$ to 7.0$)$ \\
\hline Mean total cost & 473 (186 to 760$)$ & 853 (417 to 1289$)$ & 1035 (471 to 1599$)$ \\
\hline Incremental $\operatorname{cost}^{\mathrm{b}}$ & NA & $380(-254$ to 1013$)$ & $562(-61$ to 1184$)$ \\
\hline Incremental cost per $1 \mathrm{mmHg}$ reduction in $\mathrm{SBP}^{\mathrm{a}, \mathrm{c}}$ & NA & $137^{\mathrm{b}}$ & Dominated $^{\mathrm{d}}$ \\
\hline Sensitivity analysis: ITT & $N=49$ & $N=45$ & $N=46$ \\
\hline Mean reduction in SBP & $3.3(-0.1$ to 6.7$)$ & $6.9(3.3$ to 10.4$)$ & 6.1 (2.6 to 9.7$)$ \\
\hline Incremental reduction in $\mathrm{SBP}^{\mathrm{a}, \mathrm{b}}$ & NA & $2.5(-1.9$ to 6.9$)$ & $2.6(-1.7$ to 6.9$)$ \\
\hline Mean total cost & 408 (222 to 776$)$ & 905 (450 to 1359$)$ & 1035 (585 to 1484$)$ \\
\hline Incremental cost $\mathrm{t}^{\mathrm{b}}$ & NA & $495(-135$ to 1124$)$ & $625(-1$ to 1250$)$ \\
\hline Incremental cost per $1 \mathrm{mmHg}$ reduction in $\mathrm{SBP}^{\mathrm{a}, \mathrm{c}}$ & NA & $196^{\mathrm{b}}$ & $1113^{\mathrm{d}}$ \\
\hline Secondary analysis: QALYs & $N=49$ & $N=41$ & $N=45$ \\
\hline Mean QALYs ${ }^{\mathrm{e}}$ & 0.427 & 0.422 & 0.423 \\
\hline Cost per QALY ${ }^{\mathrm{c}, \mathrm{e}}$ & NA & Dominated & Dominated \\
\hline
\end{tabular}

Data are presented as means (95\% confidence interval), costs are presented in $£$ and reductions are presented in $\mathrm{mmHg}$

$I T T$ intent to treat, NA not applicable, $Q A L Y$ quality-adjusted life-year, SBP systolic blood pressure, S-MAN self-monitoring and guided selfmanagement of anti-hypertensive medication, $S-M O N$ self-monitoring of BP, TAU treatment as usual

${ }^{a}$ Adjusted for baseline SBP

${ }^{\mathrm{b}}$ Compared with TAU, after adjustment for baseline SBP

${ }^{\mathrm{c}}$ Willingness-to-pay value at which the incremental net benefit was 0

${ }^{\mathrm{d}}$ Compared with S-MON

${ }^{\mathrm{e} E v a l u a t e d}$ at mean baseline utility (0.847)

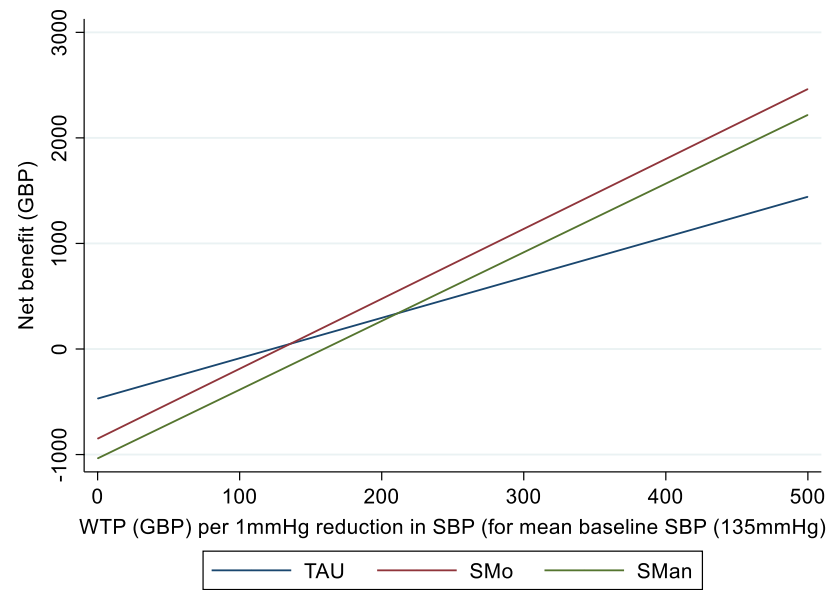

Fig. 1 Net benefit for a range of values of WTP per $1 \mathrm{mmHg}$ SBP reduction, by randomised group. $G B P$ Great British pounds, $S B P$ systolic blood pressure, SMan self-monitoring and guided self-management of anti-hypertensive medication, SMo self-monitoring of blood pressure, $T A U$ treatment as usual, $W T P$ willingness to pay

translate into sizeable risk reductions in terms of secondary stroke prevention [1]. Therefore, the relatively small reductions observed in the TEST-BP trial may convert into larger benefits in the longer term. Health economic modelling work

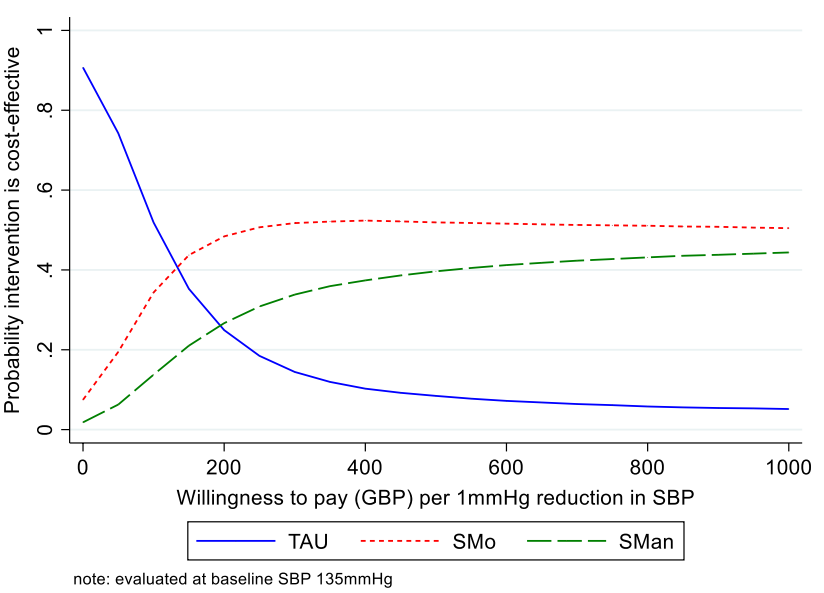

Fig. 2 Cost-effectiveness acceptability curves based on SBP reduction, by randomised group. $G B P$ Great British pounds, $S B P$ systolic blood pressure, SMan self-monitoring and guided self-management of anti-hypertensive medication, SMo self-monitoring of BP, TAU treatment as usual

would be needed to formally assess the long-term cost effectiveness of these interventions in patients with stroke or TIA, though this would necessarily require a range of assumptions regarding the conversion of reductions in short-term SBP into lifetime QALYs, as has been implemented in the 


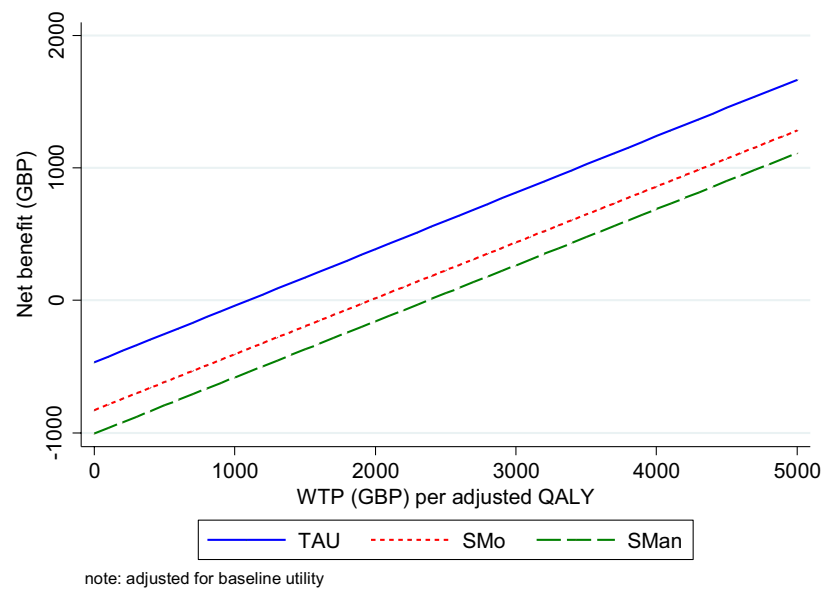

Fig. 3 Net benefit for a range of values of WTP per QALY, by randomised group. $G B P$ Great British pounds, $Q A L Y$ quality-adjusted life-year, SMan self-monitoring and guided self-management of antihypertensive medication, SMo self-monitoring of blood pressure, $T A U$ treatment as usual, WTP willingness to pay

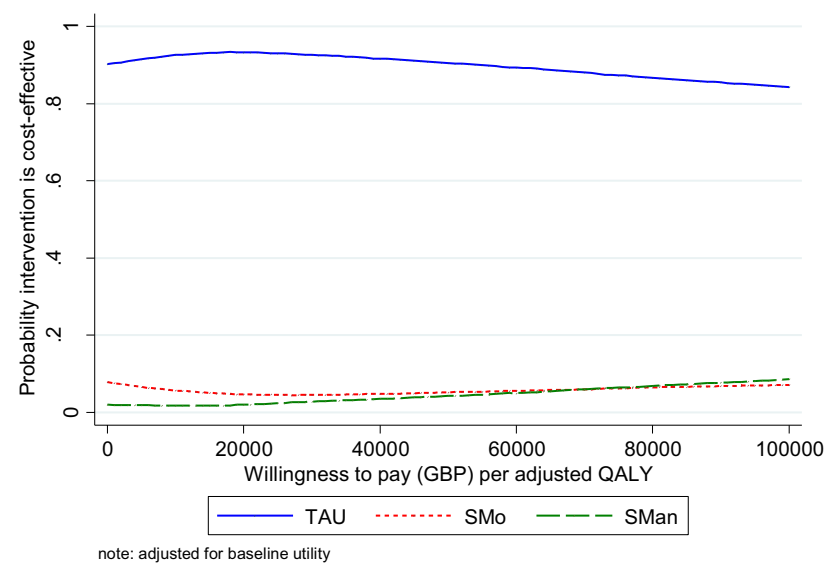

Fig. 4 Cost-effectiveness acceptability curves based on QALYs, by randomised group. $G B P$ Great British pounds, $Q A L Y$ quality-adjusted life-year, SMan self-monitoring and guided self-management of antihypertensive medication, SMo self-monitoring of BP, TAU treatment as usual

general hypertensive population [22]. It should be noted that studies of 12-24 months' duration tend to report a sustained effect from self-monitoring, but other data suggest this may diminish over the longer term [21].

Another limitation of our analysis is potential overestimation of the cost of BP monitors. A monitor is likely to last more than the 6-month time horizon of this study. However, amortising the purchase price over a longer period would not materially change our results: S-MAN would still be dominated by S-MON, and the ICER of S-MON versus TAU would be lower (more cost effective).
Finally, we reported per-protocol analyses as our primary analysis because this represents costs and outcomes for patients who actually completed the interventions, but this is of course at risk of bias. We therefore also reported the ITT analysis.

Despite the growing body of evidence demonstrating the effectiveness of self-monitoring BP in various populations, few studies have carried out economic evaluations of this approach to date. Kaambwa et al. [22] reported a long-term cost-effectiveness analysis of the TASMINH2 trial, which examined the use of telemonitoring and selfmonitoring of BP measurements in patients with hypertension. This analysis suggested that lifetime cost effectiveness was around $£ 1600$ per QALY in men and $£ 5000$ per QALY in women based on a continued $\mathrm{BP}$ reduction of $6.4 \mathrm{mmHg}$ and $4.4 \mathrm{mmHg}$, respectively.

\section{Conclusions}

Self-management of BP is unlikely to be cost effective. A self-monitoring strategy costs an extra $£ 137$ per $1 \mathrm{mmHg}$ SBP decrease over a 6-month time horizon. Decision modelling is required to project these findings to a lifetime horizon to explore whether it may yield further benefits in terms of reduced stroke recurrence and other cardiovascular impacts.

Author contributions Concept and design of TEST-BP trial: PKM, JFP. Co-applicants: PKM and JFP (principal investigators), JFP, ECFW, ABC. Economic analysis and initial draft: LGK, ECFW. Outcomes analysis: ABC, WJD. Manuscript revision and final approval: All.

Data availability The datasets and code generated during and/or analysed during the current study are available from the corresponding author on reasonable request.

\section{Compliance with Ethical Standards}

Funding TEST-BP was funded by the National Institute for Health Research (NIHR) Research for Patient Benefit Programme (RfPB; grant reference PB-PG-0909-20246). This paper presents independent research funded by the NIHR under the RfPB. The views expressed are those of the authors and not necessarily those of the NHS, the NIHR or the Department of Health.

Conflict of interest AC, WD, LK, PM and JP have no conflicts of interest that are directly relevant to the content of this article. EW has received travel expenses from the funder to attend study meetings.

Ethical approval Ethical approval for the trial was granted by the Research Ethics Committee East of England-Norfolk (ref: 11/ EE/0147), and the study was performed in accordance with the ethical standards of the Declaration of Helsinki. All participants provided written informed consent.

Open Access This article is licensed under a Creative Commons Attribution-NonCommercial 4.0 International License, which permits any 
non-commercial use, sharing, adaptation, distribution and reproduction in any medium or format, as long as you give appropriate credit to the original author(s) and the source, provide a link to the Creative Commons licence, and indicate if changes were made. The images or other third party material in this article are included in the article's Creative Commons licence, unless indicated otherwise in a credit line to the material. If material is not included in the article's Creative Commons licence and your intended use is not permitted by statutory regulation or exceeds the permitted use, you will need to obtain permission directly from the copyright holder.To view a copy of this licence, visit http://creativecommons.org/licenses/by-nc/4.0/.

\section{References}

1. Katsanos AH, Filippatou A, Manios E, Deftereos S, Parissis J, Frogoudaki A, et al. Blood pressure reduction and secondary stroke prevention: a systematic review and metaregression analysis of randomized clinical trials. Hypertension. 2017;69(1):171-9. https://doi.org/10.1161/HYPERTENSIONAHA.116.08485 (Epub 2016 Oct 31).

2. Zonneveld TP, Richard E, Vergouwen MD, Nederkoorn PJ, de Haan R, Roos YB, et al. Blood pressure-lowering treatment for preventing recurrent stroke, major vascular events, and dementia in patients with a history of stroke or transient ischaemic attack. Cochrane Database Syst Rev. 2018;7:CD007858. https://doi. org/10.1002/14651858.cd007858.pub2.

3. Rashid P, Leonardi-Bee J, Bath P. Blood pressure reduction and secondary prevention of stroke and other vascular events: a systematic review. Stroke. 2003;34(11):2741-8. https://doi. org/10.1161/01.STR.0000092488.40085.15 (Epub 2003 Oct 23).

4. Bosworth HB, Olsen MK, Grubber JM, Neary AM, Orr MM, Powers BJ, et al. Two self-management interventions to improve hypertension control: a randomized trial. Ann Intern Med. 2009;151(10):687-95. https://doi.org/10.7326/0003-4819-15110-200911170-00148.

5. McManus RJ, Mant J, Bray EP, Holder R, Jones MI, Greenfield $\mathrm{S}$, et al. Telemonitoring and self-management in the control of hypertension (TASMINH2): a randomised controlled trial. Lancet. 2010;376(9736):163-72. https://doi.org/10.1016/S0140 -6736(10)60964-6 (Epub 2010 Jul 8).

6. Tucker KL, Sheppard JP, Stevens R, Bosworth HB, Bove A, Bray $\mathrm{EP}$, et al. Self-monitoring of blood pressure in hypertension: a systematic review and individual patient data meta-analysis. PLoS Med. 2017;14(9):e1002389. https://doi.org/10.1371/journal.pmed (eCollection 2017 Sep).

7. Davison WJ, Myint PK, Clark AB, Kim LG, Wilson EC, Langley $\mathrm{M}$, et al. Does self-monitoring and self-management of blood pressure after stroke or transient ischemic attack improve control? TEST-BP, a randomized controlled trial. Am Heart J. 2018;203:105-108. https://doi.org/10.1016/j.ahj.2018.06.002 (epub Jul 27).

8. Cohen JB, Lotito MJ, Trivedi UK, Denker MG, Cohen DL, Townsend RR. Cardiovascular events and mortality in white coat hypertension: a systematic review and meta-analysis. Ann Intern Med. 2019;170(12):853-62.

9. Dolan P. Modeling valuations for EuroQol health states. Med Care. 1997;35(11):1095-108.

10. Manca A, Hawkins N, Sculpher MJ. Estimating mean QALYs in trial-based cost-effectiveness analysis: the importance of controlling for baseline utility. Health Econ. 2005;14(5):487-96. https://doi.org/10.1002/hec.944.

11. Curtis L, Burns A. Unit costs of health and social care 2018. Canterbury: University of Kent; 2018.

12. Department of Health. National schedule of reference costs 201718 2019. https://improvement.nhs.uk/resources/national-cost-colle ction/. Accessed 5 Feb 2020.

13. Department of Health. Hospital and community health series. Pay and prices inflation series 2015/6. http://www.info.doh.gov. uk/doh/finman.nsf/af3d43e36a4c8f8500256722005b77f8/360a4 7827991d10a80258036002d8d9f/\%24FILE/2015.16\%20Pay $\% 20 \% 26 \% 20$ Price\%20series.xlsx. Accessed 5 Feb 2020.

14. NHS Business Services Authority. NHS electronic drug tariff London. 2015. http://www.drugtariff.nhsbsa.nhs.uk/\#/00446515DC_2/DC00446029/Part\%20VIIIA\%20products\%20C. Accessed 5 Feb 2020.

15. National Institute for Health and Care Excellence. British National Formulary. https://bnf.nice.org.uk. Accessed 5 Feb 2020.

16. Verberk WJ, Kroon AA, Lenders JW, Kessels AG, van Montfrans GA, Smit AJ, et al. Self-measurement of blood pressure at home reduces the need for antihypertensive drugs: a randomized, controlled trial. Hypertension. 2007;50(6):1019-25. https://doi. org/10.161/HYPERTENSIONAHA.107.094193 (Epub 2007 Oct 15).

17. Omboni S, Gazzola T, Carabelli G, Parati G. Clinical usefulness and cost effectiveness of home blood pressure telemonitoring: meta-analysis of randomized controlled studies. J Hypertens. 2013;31(3):455-67. https://doi.org/10.1097/hjh.0b013e32835ca8d d (discussion 67-8).

18. McManus RJ, Mant J, Haque MS, Bray EP, Bryan S, Greenfield $\mathrm{SM}$, et al. Effect of self-monitoring and medication self-titration on systolic blood pressure in hypertensive patients at high risk of cardiovascular disease: the TASMIN-SR randomized clinical trial. JAMA. 2014;312(8):799-808. https://doi.org/10.1001/ jama.2014.10057.

19. Kerry SM, Markus HS, Khong TK, Cloud GC, Tulloch J, Coster $\mathrm{D}$, et al. Home blood pressure monitoring with nurse-led telephone support among patients with hypertension and a history of stroke: a community-based randomized controlled trial. CMAJ. 2013;185(1):23-31. https://doi.org/10.1503/cmaj.120832 (Epub 2012 Nov 5).

20. Stergiou GS, Parati G, Vlachopoulos C, Achimastos A, Andreadis E, Asmar R, et al. Methodology and technology for peripheral and central blood pressure and blood pressure variability measurement: current status and future directions-position statement of the European Society of Hypertension Working Group on blood pressure monitoring and cardiovascular variability. J Hypertens. 2016;34(9):1665-77. https://doi.org/10.1097/HJH.0000000000 000969.

21. Margolis KL, Asche SE, Dehmer SP, Bergdall AR, Green BB, Sperl-Hillen JM, et al. Long-term outcomes of the effects of home blood pressure telemonitoring and pharmacist management on blood pressure among adults with uncontrolled hypertension: follow-up of a cluster randomized clinical trial. JAMA Netw Open. 2018;1(5):e181617.

22. Kaambwa B, Bryan S, Jowett S, Mant J, Bray EP, Hobbs FD, et al. Telemonitoring and self-management in the control of hypertension (TASMINH2): a cost-effectiveness analysis. Eur J Prev Cardiol. 2014;21(12):1517-30. https://doi. org/10.177/2047487313501886 (Epub 2013 Aug 29). 